\title{
High-rank attack on HMFEv
}

\author{
Yasufumi Hashimoto ${ }^{1}$ \\ ${ }^{1}$ Department of Mathematical Sciences, University of the Ryukyus, 1 Senbaru, Nishihara-cho, \\ Nakagami-gun, Okinawa, Japan \\ E-mail hashimoto@math.u-ryukyu.ac.jp
}

Received October 18, 2017, Accepted February 19, 2018

\begin{abstract}
HMFEv is a new multivariate signature scheme proposed at PQCrypto 2017. This is a vinegar variant of multi-HFE (Chen et al., 2008). While the original multi-HFE is known to be insecure against the direct attack (Huang et al., 2015), the min-rank attack (Bettale et al., 2013) and the attack using a diagonalization approach (Hashimoto, 2017), HMFEv is considered to be secure enough against these attacks. However, the security against the high-rank attack had not been studied at all. In the present paper, we study the structure of HMFEv and discuss its security against the high-rank attack.
\end{abstract}

Keywords HMFEv, high-rank attack, multivariate public key cryptosystem (MPKC), postquantum cryptography

Research Activity Group Algorithmic Number Theory and Its Applications

\section{Introduction}

The multi-HFE [1] is one of public key cryptosystems whose public keys are sets of multivariate quadratic forms over finite fields. The quadratic forms in multiHFE are generated by a set of multivariate quadratic forms over an extension field of the basic field. Unfortunately, the multi-HFE is known to be insecure against the direct attack [2], the min-rank attack [3] and the attack using a diagonalization approach [4].

Recently in PQCrypto 2017, a vinegar variant of multi-HFE, called HMFEv, was proposed by Petzoldt et al. [5]. This vinegar variant succeeds to enhance the security against the known attacks [2-4] and then HMFEv had been expected to be one of signature schemes, secure and efficient enough under suitable parameter selections [5] (see Table 1). However, the security against the high-rank attack had not been studied yet at all.

In this paper, we study the structure of HMFEv and discuss the security of HMFEv against the high-rank attack. Based on the results of our experiments given in Table 2, we can conclude that the security of HMFEv is much less than expected.

\section{Multi-HFE and HMFEv}

In this section, we describe the constructions of multiHFE [1] and HMFEv [5].

\subsection{Multi-HFE}

Let $n, N, r \geq 1$ be integers with $n=N r$ and $q$ a power of prime. Denote by $k$ a finite field of order $q$ and $K$ an $r$ extension of $k$. Define the quadratic map $\mathcal{G}: K^{N} \rightarrow K^{N}$ by

$$
\begin{aligned}
& X=\left(X_{1}, \ldots, X_{N}\right)^{t} \\
& \mathcal{G}(X)=\left(\mathcal{G}_{1}(X), \ldots, \mathcal{G}_{N}(X)\right)^{t} \\
& \mathcal{G}_{l}(X)=\sum_{1 \leq i \leq j \leq N} \alpha_{i j}^{(l)} X_{i} X_{j}+\sum_{1 \leq i \leq N} \beta_{i}^{(l)} X_{i}+\gamma^{(l)}
\end{aligned}
$$

for $1 \leq l \leq N$, where $\alpha_{i j}^{(l)}, \beta_{i}^{(l)}, \gamma^{(l)} \in K$.

The secret key is a pair of two invertible affine maps $S, T: k^{n} \rightarrow k^{n}$ and the public key is the quadratic map $F: k^{n} \rightarrow k^{n}$ defined by $F:=T \circ \phi_{N}^{-1} \circ \mathcal{G} \circ \phi_{N} \circ S$, where $\phi_{N}: k^{n} \rightarrow K^{N}$ is a one-to-one map.

In the multi-HFE, a plain-text $p \in k^{n}$ is encrypted by $c=F(p) \in k^{n}$, and the cipher-text $c \in k^{n}$ is decrypted as follows. First, compute $Z=\left(Z_{1}, \ldots, Z_{N}\right)^{t}:=$ $\phi_{N}\left(T^{-1}(c)\right) \in K^{N}$. Next, find a common solution $X \in$ $K^{N}$ of the equations

$$
\mathcal{G}_{1}(X)=Z_{1}, \quad \ldots, \quad \mathcal{G}_{N}(X)=Z_{N}
$$

The plain-text $p \in k^{n}$ is given by $S^{-1}\left(\phi_{N}^{-1}(X)\right)$.

To find $X$ with (1), one needs to solve a system of $N$ quadratic equations of $N$ variables. Since the complexity of solving it is in exponential for $N$ (see e.g. [6,7]), the number $N$ can not be taken large.

\section{2 $\mathrm{HMFEv}$}

Let $n, m, N, r, v \geq 1$ be integers with $m:=N r, n:=$ $m+v$ and $q$ a power of prime. Denote by $k$ the finite field of order $q$ and $K$ the $r$-extension of $k$. Define the $\operatorname{map} \mathcal{G}: K^{N} \times k^{v} \rightarrow K^{N}$ by $X=\left(X_{1}, \ldots, X_{N}\right)^{t}, \quad u=\left(u_{1}, \ldots, u_{v}\right)^{t}$,

$\mathcal{G}(X, u)=\left(\mathcal{G}_{1}(X, u), \ldots, \mathcal{G}_{N}(X, u)\right)^{t}$,

$\mathcal{G}_{l}(X, u)=\sum_{1 \leq i \leq j \leq N} \alpha_{i j}^{(l)} X_{i} X_{j}+\sum_{1 \leq i \leq N} \beta_{i}^{(l)}(u) X_{i}+\gamma^{(l)}(u)$

for $1 \leq l \leq N$, where $\alpha_{i j}^{(l)} \in K, \beta_{i}^{(l)}: k^{v} \rightarrow K$ is an affine form and $\gamma^{(l)}: k^{v} \rightarrow K$ is a quadratic form.

The secret key is a pair of two invertible affine maps $S: k^{n} \rightarrow k^{n}, T: k^{m} \rightarrow k^{m}$ and the public key is the quadratic map $F: k^{n} \rightarrow k^{m}$ defined by

$$
F:=T \circ \phi_{N}^{-1} \circ \mathcal{G} \circ \phi_{N, v} \circ S,
$$

where $\phi_{N}: k^{m} \rightarrow K^{N}, \phi_{N, v}: k^{n} \rightarrow K^{N} \times k^{v}$ are one- 
Table 1. Parameter Selection of HMFEv [5].

\begin{tabular}{|r|rr|rrr|r|}
\hline$q$ & $n$ & $m$ & $N$ & $r$ & $v$ & Security \\
\hline \hline 31 & 44 & 36 & 2 & 18 & 8 & 80 bit \\
256 & 39 & 27 & 3 & 9 & 12 & 80 bit \\
\hline 31 & 68 & 56 & 2 & 28 & 12 & 128 bit \\
256 & 61 & 45 & 3 & 15 & 16 & 128 bit \\
\hline 31 & 97 & 80 & 2 & 40 & 17 & 192 bit \\
256 & 90 & 69 & 3 & 23 & 21 & 192 bit \\
\hline 31 & 131 & 110 & 2 & 55 & 21 & 256 bit \\
256 & 119 & 93 & 3 & 31 & 26 & 256 bit \\
\hline
\end{tabular}

\section{to-one maps.}

In the signature scheme HMFEv, a given message $y \in k^{m}$ is signed as follows. First, compute $Z=$ $\left(Z_{1}, \ldots, Z_{N}\right)^{t}:=\phi_{N}\left(T^{-1}(y)\right)$ and choose $u \in k^{v}$. Next, find a common solution $X \in K^{N}$ of the equations

$$
\mathcal{G}_{1}(X, u)=Z_{1}, \quad \ldots, \quad \mathcal{G}_{N}(X, u)=Z_{N} .
$$

The signature for $y \in k^{m}$ is $S^{-1}\left(\phi_{N, v}^{-1}(X, u)\right)$. The signature $x \in k^{n}$ is verified if $F(x)=y$ holds.

To find $X$ with (3), one needs to solve a system of $N$ quadratic equations of $N$ variables. Then, similar to the multi-HFE, the number $N$ cannot be large since the complexity of solving it is exponential for $N$. In [5] (see Table 1), Petzeldt et al. selected the parameters of HMFEv with $N=2,3$ as a signature scheme secure and efficient enough for practical use.

We note that the constant parts of $S, T$ do not contribute to enhance the security. In fact, for $s \in k^{n}$, $t \in k^{m}, S_{0}(x):=x+s$ and $T_{0}(y):=y+t$, the map $\phi_{N} \circ T_{0} \circ G \circ S_{0} \circ \phi_{N, v}^{-1}$ is also a quadratic map similar to (2). Then we can consider that $S, T$ are linear maps without loss of generality.

\section{Security analysis}

In this section, we study the structure of HMFEv and discuss the security against the rank attacks. We first study the structures of polynomials in HMFEv.

\subsection{Polynomials in HMFEv}

For integers $n_{1}, n_{2} \geq 1$ and a finite field $k$, let $\mathrm{M}_{n_{1}, n_{2}}(k)$ be the set of $n_{1} \times n_{2}$ matrices of $k$-entries. Denote by $I_{n} \in \mathrm{M}_{n, n}(k)$ the identity matrix and by $0_{n_{1}, n_{2}} \in \mathrm{M}_{n_{1}, n_{2}}(k)$ the zero matrix. For simplicity, we write $\mathrm{M}_{n}(k):=\mathrm{M}_{n, n}(k)$ and $0_{n}:=0_{n, n}$. For an integer $l \geq 1$ and a matrix $A=\left(a_{i j}\right)_{i, j}$, we denote by $A^{(l)}:=\left(a_{i j}^{l}\right)_{i, j}$.

Let $\left\{\theta_{1}, \ldots, \theta_{r}\right\} \subset K$ be a basis of $K$ over $k$ and define $\Theta_{N}:=\left(\theta_{j}^{q^{i-1}} \cdot I_{N}\right)_{1 \leq i, j \leq r} \in \mathrm{M}_{m}(K)$ and $\Theta_{N, v}:=$ $\left(\begin{array}{cc}\Theta_{N} & \\ & I_{v}\end{array}\right) \in \mathrm{M}_{n}(K)$. The one-to-one maps $\phi_{N}: k^{m} \rightarrow$ $K^{N}$ and $\phi_{N, v}: k^{n} \rightarrow K^{N} \times k^{v}$ are given by the matrices $\Theta_{N}$ and $\Theta_{N, v}$ respectively. In fact, it holds

$$
\phi_{N}=\psi_{N}^{-1} \circ \Theta_{N}, \quad \phi_{N, v}=\psi_{N, v}^{-1} \circ \Theta_{N, v}
$$

for the two maps $\psi_{N}: K^{N} \rightarrow K^{N r}, \psi_{N, v}: K^{N} \times k^{v} \rightarrow$ $K^{N r} \times k^{v}$ defined by

$$
\begin{aligned}
& \psi_{N}\left(\alpha_{1}, \ldots, \alpha_{N}\right)=\left(\alpha_{1}, \ldots, \alpha_{N}, \alpha_{1}^{q}, \ldots, \ldots, \alpha_{N}^{q^{r-1}}\right)^{t}, \\
& \psi_{N, v}\left(\alpha_{1}, \ldots, \alpha_{N}, u_{1}, \ldots, u_{v}\right) \\
& \quad=\left(\alpha_{1}, \ldots, \alpha_{N}, \alpha_{1}^{q}, \ldots, \ldots, \alpha_{N}^{q^{r-1}}, u_{1}, \ldots, u_{v}\right)^{t} .
\end{aligned}
$$

Then the public key $F$ is described by

$$
F=\left(T \circ \Theta_{N}^{-1}\right) \circ\left(\psi_{N} \circ \mathcal{G} \circ \psi_{N, v}^{-1}\right) \circ\left(\Theta_{N, v} \circ S\right),
$$

namely

$$
\begin{aligned}
F(x)= & \left(f_{1}(x), \ldots, f_{m}(x)\right)^{t} \\
= & \left(T \circ \Theta_{N}^{-1}\right) \cdot\left(\mathcal{G}_{1}\left(\phi_{N, v}(S(x))\right), \ldots,\right. \\
& \mathcal{G}_{N}\left(\phi_{N, v}(S(x))\right), \mathcal{G}_{1}\left(\phi_{N, v}(S(x))\right)^{q}, \ldots, \\
& \left.\mathcal{G}_{N}\left(\phi_{N, v}(S(x))\right)^{q^{r-1}}\right)^{t} .
\end{aligned}
$$

When we express the polynomials $\mathcal{G}_{1}, \ldots, \mathcal{G}_{N}$ by

$$
\mathcal{G}_{l}(X, u)=\left(X^{t}, u^{t}\right)\left(\begin{array}{ll}
A_{l} & B_{l} \\
B_{l}^{t} & C_{l}
\end{array}\right)\left(\begin{array}{c}
X \\
u
\end{array}\right)+(\text { linear form })
$$

as quadratic forms of $X, u$ with matrices $A_{l} \in \mathrm{M}_{N}(K)$, $B_{l} \in \mathrm{M}_{N, v}(K), C_{l} \in \mathrm{M}_{v}(K)$, the polynomials $\mathcal{G}_{1}(X, u)$, $\ldots, \mathcal{G}_{N}(X, u), \mathcal{G}_{1}(X, u)^{q}, \ldots, \ldots, \mathcal{G}_{N}(X, u)^{q^{r-1}}$ in (4) are written as quadratic polynomials of

$$
\begin{aligned}
\bar{X} & :=\psi_{N, v}(X, u) \\
& =\left(X_{1}, \ldots, X_{N}, X_{1}^{q}, \ldots, \ldots, X_{N}^{q^{r-1}}, u_{1}, \ldots, u_{v}\right)^{t}
\end{aligned}
$$

in the forms

$$
\begin{aligned}
& \mathcal{G}_{l}(X, u)=\bar{X}^{t}\left(\begin{array}{cc|c}
A_{l} & & B_{l} \\
\hline B_{l}^{t} & 0_{m-N} & \\
\hline
\end{array}\right. \\
& +(\text { linear form of } \bar{X}) \text {, } \\
& \mathcal{G}_{l}(X, u)^{q}=\bar{X}^{t}\left(\begin{array}{ccc|c}
0_{N} & & & B_{l}^{(q)} \\
& A_{l}^{(q)} & & 0_{m-2 N} \\
\hline & B_{l}^{(q)^{t}} & & C_{l}^{(q)}
\end{array}\right) \bar{X} \\
& +(\text { linear form of } \bar{X}) \text {, } \\
& \mathcal{G}_{l}(X, u)^{q^{r-1}}=\bar{X}^{t}\left(\begin{array}{cc|c}
0_{m-N} & \\
& A_{l}^{\left(q^{r-1}\right)} & B_{l}^{\left(q^{r-1}\right)} \\
\hline B_{l}^{\left(q^{r-1}\right)^{t}} & C_{l}^{\left(q^{r-1}\right)}
\end{array}\right) \bar{X} \\
& +(\text { linear form of } \bar{X}) \text {. }
\end{aligned}
$$

This means that the quadratic forms in the public key are expressed by

$$
f_{l}(x)=x^{t} F_{l} x+(\text { linear form of } x),
$$

where

$$
F_{l}=\left(\Theta_{N, v} S\right)^{t}\left(\begin{array}{ccc|c}
*_{N} & & & * \\
& \ddots & & \vdots \\
& & *_{N} & * \\
\hline * & \cdots & * & *_{v}
\end{array}\right)\left(\Theta_{N, v} S\right) .
$$

In the next two subsections, we discuss the security of HMFEv against the rank attacks based on these facts.

\subsection{Min-rank attack}

Let $F_{1}, \ldots, F_{m}$ be the coefficient matrices of the quadratic forms $f_{1}(x), \ldots, f_{m}(x)$ respectively. The min-rank attack, introduced by Kipnis-Shamir [8] and developed by Bettale et al. [3], is an attack to recover $T$ (partially) by finding $\alpha_{1}, \ldots, \alpha_{m} \in K$ such that the rank of $H:=\alpha_{1} F_{1}+\cdots+\alpha_{m} F_{m}$ is at most $R$ if there exist such $\alpha_{1}, \ldots, \alpha_{m} \in K$ and an integer $1 \leq R<n$. For HMFEv, due to (4) and (5), we see that there exist such $\alpha_{1}, \ldots, \alpha_{m} \in K$ with $R=N+v$ and $H$ is one of the 
following forms with high probability.

$$
\begin{aligned}
& \left(\Theta_{N, v} S\right)^{t}\left(\begin{array}{rrr}
*_{N} & & * \\
& 0_{m-N} & \\
* & & *_{v}
\end{array}\right)\left(\Theta_{N, v} S\right), \\
& \left(\Theta_{N, v} S\right)^{t}\left(\begin{array}{rrr}
0_{N} & & \\
& *_{N} & * \\
* & 0_{m-2 N} & *_{v}
\end{array}\right)\left(\Theta_{N, v} S\right), \\
& \ldots,\left(\Theta_{N, v} S\right)^{t}\left(\begin{array}{rrr}
0_{m-N} & \\
& *_{N+v}
\end{array}\right)\left(\Theta_{N, v} S\right) .
\end{aligned}
$$

Once such a matrix $H$ is given, the attacker can recover keys equivalent to $(S, T)$ easily (see [3]).

To find such $\alpha_{1}, \ldots, \alpha_{m} \in K$, the attacker generates a system of polynomial equations of $m$ variables $z_{1}, \ldots, z_{m}$ derived from the condition that the rank of $H\left(z_{1}, \ldots, z_{m}\right):=z_{1} F_{1}+\cdots+z_{m} F_{m}$ is at most $N+v$ and solve it by, e.g., the Gröbner basis algorithm. Since the condition that the rank of $A$ is at most $R$ is equivalent that the determinants of arbitrary $(R+1) \times(R+1)$ minor matrices in $A$ are zero, the min-rank attack requires to solve a system of polynomial equations of degree (at most) $N+v+1$ and of $m$ variables. Based on the result in [3], the authors in [5] claimed that the complexity of the min-rank attack is $O\left(\left(\begin{array}{c}m+N+v+1 \\ N+v+1\end{array}\right)\right.$ w where $2 \leq w<3$ is an exponent of the Gaussian elimination. This means that, if one takes $v$ sufficiently large, HMFEv is secure enough against the min-rank attack.

\subsection{High-rank attack}

The high-rank attack, introduced in $[9,10]$, is to find $\beta_{1}, \ldots, \beta_{L} \in K$ such that the rank of $P:=F_{m}+\beta_{1} F_{1}+$ $\cdots+\beta_{L} F_{L}$ is at most $R$ if there exist such integers $1 \leq L, R<n$ and $\beta_{1}, \ldots, \beta_{L} \in K$. For HMFEv, recall that $F_{1}, \ldots, F_{m}$ are as written in (6). Due to (4) and (5), we see that the first $N$ columns and lows of the central matrix in (6) are derived from linear sums of $N$ polynomials $\mathcal{G}_{1}(X, u), \ldots, \mathcal{G}_{N}(X, u)$. Then, removing the contributions of such $N$ polynomials, we can get a matrix of rank at most $n-N$. This means that the high-rank attack is available on HMFEv with $(L, R)=(N, n-N)$. We now describe how to recover an equivalent key of HMFEv.

Input. The public matrices $F_{1}, \ldots, F_{m}$.

Output. Invertible matrices $S^{\prime} \in \mathrm{M}_{n}(k), T^{\prime} \in \mathrm{M}_{m}(k)$ such that $\phi_{N} \circ T^{\prime} \circ F \circ S^{\prime} \circ \phi_{N, v}^{-1}: K^{N} \times k^{v} \rightarrow K^{N}$ is a quadratic map similar to $(2)$.

Step 1. Find $\beta_{1}, \ldots, \beta_{N} \in K$ such that $P:=F_{m}+$ $\beta_{1} F_{1}+\cdots+\beta_{N} F_{N}$.

Step 2. Find a matrix $Q \in \mathrm{M}_{n, N}(K)$ with $P Q=0_{n, N}$. Step 3. Choose $Q_{0} \in \mathrm{M}_{n, v}(k)$ randomly and put $\tilde{Q}:=\left(Q, Q^{(q)}, \ldots, Q^{\left(q^{r-1}\right)}, Q_{0}\right) \in \mathrm{M}_{n}(k) \Theta_{N, v}^{-1}$. If $\tilde{Q}$ is not invertible, change $Q, Q_{0}$. Compute $F_{l}^{\prime}:=\tilde{Q}^{t} F_{l} \tilde{Q}$ for $1 \leq l \leq m$.

Step 4. Find a matrix $W=\left(w_{i j}\right)_{i, j} \in \mathrm{M}_{N, m}(K)$ with

$$
F_{i}^{\prime \prime}:=\sum_{1 \leq j \leq m} w_{i j} F_{j}^{\prime}=\left(\begin{array}{lll}
*_{N} & & * \\
& 0_{m-N} & \\
* & & *_{v}
\end{array}\right)
$$

for $1 \leq i \leq N$. Put $\tilde{W}:=\left(\begin{array}{c}W \\ W^{(q)} \\ \vdots \\ W^{\left(q^{r-1}\right)}\end{array}\right) \in \Theta_{N} \mathrm{M}_{m}(k)$. If
$\tilde{W}$ is not invertible, change $W$.

Step 5. Output $S^{\prime}:=\tilde{Q} \Theta_{N, v}$ and $T^{\prime}:=\Theta_{N}^{-1} \tilde{W}$.

We explain why this attack recovers an equivalent key. As discussed before, there exist $\beta_{1}, \ldots, \beta_{N} \in K$ in Step 1 and we can easily check that $P$ is one of the following forms with high probability.

$$
\begin{aligned}
& \left(\Theta_{N, v} S\right)^{t}\left(\begin{array}{cc}
0_{N} & \\
& *_{n-N}
\end{array}\right)\left(\Theta_{N, v} S\right), \\
& \left(\Theta_{N, v} S\right)^{t}\left(\begin{array}{ccc}
*_{N} & & * \\
& 0_{N} & \\
* & & *_{n-2 N}
\end{array}\right)\left(\Theta_{N, v} S\right), \\
& \cdots,\left(\Theta_{N, v} S\right)^{t}\left(\begin{array}{lll}
*(r-1) N & & * \\
* & 0_{N} & \\
* & & *_{v}
\end{array}\right)\left(\Theta_{N, v} S\right) .
\end{aligned}
$$

Since $P$ is of rank at most $n-N$, there exists a matrix $Q$ in Step 2 and it can be found by linear operations. Due to $(7)$, we see that a matrix $\tilde{Q}=(Q, *)$ satisfies $\tilde{Q}^{t} P \tilde{Q}=\left(\begin{array}{ll}0_{N} & \\ & *\end{array}\right)$ and then $\left(\Theta_{N, v} S\right) \tilde{Q}$ is in the form $\left(\begin{array}{cc}*_{N} & * \\ 0 & *\end{array}\right)$ or its permutation. Lemma 3.2 in [4] tells that the matrix $\tilde{Q}$ in Step 3 is in $\mathrm{M}_{n}(k) \Theta_{N, v}^{-1}$ and $\left(\Theta_{N, v} S\right) \tilde{Q} \in \Theta_{N, v} \mathrm{M}_{n}(k) \Theta_{N, v}^{-1}$. Thus $\left(\Theta_{N, v} S\right) \tilde{Q}$ must be $\left(\begin{array}{ccc|c}*_{N} & & & * \\ & \ddots & & \vdots \\ & & *_{N} & * \\ \hline & & & *_{v}\end{array}\right)$ or its permutation. This means, due to $(6)$, that $F_{l}^{\prime}=\left(\begin{array}{ccc|c}*_{N} & & & * \\ & \ddots & & \vdots \\ & & *_{N} & * \\ \hline * & \cdots & * & *_{v}\end{array}\right)$ for $1 \leq$ $l \leq m$. Since $F_{l}^{\prime}$ is a linear sum of matrices similar to the coefficient matrices given in (5), a matrix $W$ in Step 4 exists and it is found by linear operations. The matrices $F_{1}^{\prime \prime}, \ldots, F_{N}^{\prime \prime}$ are similar to the coefficient matrices of $\mathcal{G}_{1}(X, u), \ldots, \mathcal{G}_{N}(X, u)$. We thus conclude that $\left(S^{\prime}, T^{\prime}\right)$ in Step 5 is an equivalent key.

It is easy to see that Step 2-5 require only linear operations. Then the complexity of Step 1 is important in this attack. To find $\beta_{1}, \ldots, \beta_{N}$ in Step 1, we state a system of polynomial equations of $N$ variables $y_{1}, \ldots, y_{N}$ derived from the condition that the rank of $P\left(y_{1}, \ldots, y_{N}\right):=F_{m}+y_{1} F_{1}+\cdots+y_{N} F_{N}$ is at most $n-N$ and solve it by, e.g., the Gröbner basis algorithm. Since this condition is equivalent that the determinants of arbitrary $(n-N+1) \times(n-N+1)$-minor matrices of $P\left(y_{1}, \ldots, y_{N}\right)$ are zero, the attacker needs to solve a system of polynomial equations of degree at most $n-N+1$ and of $N$ variables. Then we can consider that the complexity of the high-rank attack on HMFEv highly depends on $N$ and the contribution of $v$ for the security seems not too much.

Note that, for integers $M_{1}, N_{1}, d \geq 1$ with $M_{1} \geq N_{1}$ and a semi-regular system of polynomials $\left\{p_{1}(x), \ldots, p_{M_{1}}(x)\right\}$ of $N_{1}$-variables and of degree $d$, it is known (e.g. [6,7]) that the complexity of the Gröbner basis algorithm on this polynomial system is bounded by $O\left(\left(\begin{array}{c}N_{1}+d_{\text {reg }} \\ N_{1}\end{array}\right)^{w}\right)$, where $d_{\text {reg }}$ is a constant, called the degree of regularity, given by the index of the first non- 
Table 2. Running times of high-rank attack on HMFEv.

\begin{tabular}{|r|rr|rrr||rr|}
\hline$q$ & $n$ & $m$ & $N$ & $r$ & $v$ & Time & (Security) \\
\hline \hline 31 & 44 & 36 & 2 & 18 & 8 & $2.20 \mathrm{~s}$ & (80bit) \\
256 & 39 & 27 & 3 & 9 & 12 & $13.2 \mathrm{~s}$ & (80bit) \\
\hline 31 & 68 & 56 & 2 & 28 & 12 & $19.1 \mathrm{~s}$ & (128bit) \\
256 & 61 & 45 & 3 & 15 & 16 & $261 \mathrm{~s}$ & (128bit) \\
\hline 31 & 97 & 80 & 2 & 40 & 17 & $113 \mathrm{~s}$ & (192bit) \\
256 & 90 & 69 & 3 & 23 & 21 & - & (192bit) \\
\hline 31 & 131 & 110 & 2 & 55 & 21 & $701 \mathrm{~s}$ & (256bit) \\
256 & 119 & 93 & 3 & 31 & 26 & - & (256bit) \\
\hline
\end{tabular}

positive coefficient of the univariate polynomial $C(t):=$ $\left(1-t^{d}\right)^{M_{1}}(1-t)^{-N_{1}}$. This means that, if $M_{1}$ is sufficiently larger than $N_{1}$, we have $d_{\text {reg }}=d$. We thus expect that the complexity of the high-rank attack on HMFEv is

$$
O\left(\left(\begin{array}{c}
n+1 \\
N
\end{array}\right)^{w}\right)
$$

since $\left(N_{1}, d\right)=(N, n-N+1)$ for the polynomial system in the high-rank attack and $M_{1}$ can be taken at most $\left(\begin{array}{c}n \\ n-N+1\end{array}\right)^{2}$. Remark that (8) is the number of operations on $K$ and then the real running time seems at most $(r \log q)^{2}$ times larger than (8). While, on this paper, we avoid to give a concrete proof for the estimate (8) of the high-rank attack, we consider that (8) is not far from the real complexity of the high-rank attack.

\subsection{Experiments of the high-rank attack}

We implemented the high-rank attack on HMFEv by Magma [11] ver.2.22-3 on Windows 8.1, Core(TM)i7$4800 \mathrm{MQ}, 2.70 \mathrm{GHz}$ for the parameters in Table 1 ( [5]). In our implementation, we first choose an integer $M$ sufficiently larger than $N$, and generate $M$ equations of $N$ variables $\left(y_{1}, \ldots, y_{N}\right)$ by the determinants of $(n-N+$ $1) \times(n-N+1)$ minor matrices of $P\left(y_{1}, \ldots, y_{N}\right)$. Next, we find a common solution $\left(y_{1}, \ldots, y_{N}\right)=\left(\beta_{1}, \ldots, \beta_{N}\right)$ of such $M$ equations by the Gröbner basis algorithm. Finally, we check whether the rank of $P\left(\beta_{1}, \ldots, \beta_{N}\right)$ is at most $n-N$.

Remark that the Gaussian elimination is not efficient to compute a determinant of a polynomial matrix of large size. We then used an algorithm introduced in [12] instead of the Gaussian elimination for computations of polynomial matrices.

We also remark that, if $q$ is even, we use $F_{l}+F_{l}^{t}$ instead of the coefficient matrix $F_{l}$. The matrix $F_{l}+F_{l}^{t}$ is symmetric and skew-symmetric since the field $k$ is of even characteristic. It is known (e.g. [13]) that the determinant of a skew-symmetric matrix is zero when the size of the matrix is odd and is a square when that is even. We then have to arrange our attack based on this fact. Fortunately, the arrangement for even characteristic cases was already discussed in [3] for the min-rank attack and we can apply it also for the high-rank attack.

We describe the running times of the high-rank attack in Table 2 by taking $M=3$ for $(q, N)=(31,2)$ and $M=10$ for $(q, N)=(256,3)$. These results show that HMFEv with $N=2$ is not secure at all. While the complexities for the cases of $N=3$ is much more than the cases of $N=2$, we can consider that the security is far from $80 \sim 256$ bit. Though one requires a larger $N$ to generate a secure HMFEv, it lacks the efficiency of signature generation.

\section{Conclusion}

The signature scheme HMFEv is a vinegar variant of multi-HFE. It is known [5] that, if the vinegar parameter $v$ is larger, HMFEv against the min-rank attack is exponentially more secure. However, the security against the high-rank attack does not highly depend on $v$ and then HMFEv with the parameters selected in [5] (Table 1) is much less secure than expected. While HMFEv with larger $N$ is secure enough, it lacks the efficiency of the signature generation. We thus conclude that this scheme has a serious trade-off between the security and efficiency.

\section{Acknowledgments}

The author was supported by JST CREST no. JPMJCR14D6 and JSPS Grant-in-Aid for Scientific Research (C) no. 17K05181. He would like to thank the anonymous referee(s) for reading the previous draft carefully and giving helpful comments.

\section{References}

[1] C.H.O. Chen, M.S. Chen, J. Ding, F. Werner and B.Y. Yang, Odd-char multivariate hidden field equations, http://eprint.iacr.org/2008/543, 2008.

[2] M.D.A. Huang, M. Kosters, Y. Yang and S.L. Yeo, On the last fall degree of zero-dimensional Weil descent systems, arXiv:1505.02532 [math.AC], 2015.

[3] L. Bettale, J.C. Faugere and L. Perret, Cryptanalysis of HFE, multi-HFE and variants for odd and even characteristic, Des. Codes Cryptogr., 69 (2013), 1-52.

[4] Y. Hashimoto, Key recovery attacks on multivariate public key cryptosystems derived from quadratic forms over an extension field, IEICE Trans. Fundamentals, 100-A (2017), 1825.

[5] A. Petzoldt, M.S. Chen, J. Ding and B.Y. Yang, HMFEv - An efficient multivariate signature scheme, in: Proc. of PQCrypto 2017, T. Lange, T. Takagi eds., LNCS, Vol. 10346, pp. 205223, Springer-Verlag, Cham, 2017.

[6] M. Bardet, J.C. Faugère, B. Salvy and B.Y. Yang, Asymptotic expansion of the degree of regularity for semi-regular systems of equations, MEGA'05 (2005).

[7] L. Bettale, J.C. Faugère and L. Perret, Solving polynomial systems over finite fields: Improved analysis of the hybrid approach, in: Proc. of ISSAC 2012, J. van der Hoeven and M. van Hoeij eds., pp.67-74, ACM, New York, 2012.

[8] A. Kipnis and A. Shamir, Cryptanalysis of the HFE public key cryptosystem by relinearization, in: Proc. of CRYPTO 1999, M. Wiener eds., LNCS, Vol. 1666, pp.19-30, Springer-Verlag, Berlin, 1999.

[9] S. Hasegawa and T. Kaneko, An attacking method for a public-key cryptosystem based on the difficulty of solving a system of non-linear equations (in Japanese), in: Proc. of 10th Symposium on Information Theory and Its Applications, JA5-3, 1987.

[10] D. Coppersmith, J. Stern and S. Vaudenay, Attacks on the birational permutation signature schemes, in: Proc. of CRYPTO 1993, D.R. Stinson eds., LNCS, Vol. 773, pp.435443, Springer-Verlag, Berlin, 1994

[11] W. Bosma, J. Cannon and C. Playoust, The Magma algebra system. I. The user language. J. Symbolic Comput. 24 (1997), $235-265$.

[12] E. V. Krishnamurthy, Error-free polynomial matrix computations, Texts and Monographs in Computer Science, SpringerVerlag, New York, 1985.

[13] A. Cayley, Sur les determinants gauches (On skew determinants), J. Reine Angew. Math. 38 (1849), 93-96. 